\title{
EXCITONIC EFFECTS IN LINEAR AND PHOTOINDUCED FARADAY ROTATION IN SEMIMAGNETIC SEMICONDUCTORS
}

\author{
P. LeIsching ${ }^{a, b}$, R. PANKoKE ${ }^{a}$, C. Buss ${ }^{a}$, R. FreY ${ }^{a}$, C. FlytzANis ${ }^{a}$, \\ J. CIBERT $^{c}$ AND A. WASIELA ${ }^{c}$ \\ ${ }^{a}$ Ecole Polytechnique, Laboratoire d'Optique Quantique du C.N.R.S. \\ 91128 Palaiseau Cédex, France \\ ${ }^{6}$ Max-Born-Institute, Rudower Chaussee 6, 12489 Berlin, Germany \\ ${ }^{c}$ Lab. de Spectrométrie Physique, Université Joseph Fourier-Grenoble and C.N.R.S. \\ 38402 St. Martin d'Hères Cédex, France
}

We investigate the influence of the Coulomb interaction on the giant linear and photoinduced Faraday rotation in the bulk $\mathrm{Cd}_{1-x} \mathrm{Mn}_{x}$ Te system. Experimental results are presented and discussed by calculating the complex dielectric function. The anisotropy and $k$-dependence of the magnetic spin-exchange interaction and the Coulomb interaction between optically excited bound and unbound electron-hole pairs are considered using a microscopic theory. At high excitation densities, the excitonic nonlinearities of the $1 s$ transitions are investigated.

PACS numbers: 78.66.Hf, 78.20.Ls, 75.30.Et, 42.65.-k

The linear and photoinduced Faraday rotation in bulk and quantum-confined semimagnetic semiconductors has recently gained much interest [1-6]. Exploiting the excitonic nonlinearities and photoinduced circular birefringence may pave the way towards attractive applications in nonlinear signal processing [1].

The giant magneto-optical effects observed in $\mathrm{Cd}_{1-x} \mathrm{Mn}_{x} \mathrm{Te}$ materials are due to the exchange interaction between the spins of the $3 d^{5}$ electrons of $\mathrm{Mn}^{2+}$ and the spins of the carriers in the valence- and conduction-bands [2]. We discuss the strong effects of the Coulomb enhancement to the observed linear Faraday rotation angle for both bound (excitonic) and unbound (free) interband transitions and demonstrate the dominance of the excitonic transitions in the linear and photoinduced Faraday rotation at $5 \mathrm{~K}$. For the first time, a photoinduced blue shift of the $1 s$ excitonic transition in a semiconductor bulk material is reported.

The zero field transmission characterization of the linear optical properties at $5 \mathrm{~K}$ of a $4000 \AA$ thick $\mathrm{Cd}_{0.95} \mathrm{Mn}_{0.05} \mathrm{Te}$ layer reveals a pronounced $1 s$ excitonic peak followed by a broadened $2 s / n s$ transition and the continuum edge [3]. Applying a magnetic field, the spin-exchange interaction leads to a strong splitting of the $\sigma_{ \pm 3 / 2}$ transitions and much smaller splitting of the $\sigma_{ \pm 1 / 2}$ transitions with relative 
intensities of 3:1 [2]. The resulting linear Faraday rotation spectrum close to the band edge at $0.5 \mathrm{~T}$ is depicted in Fig. 1a. Increasing the field up to $2 \mathrm{~T}$ allows the distinct observation of further excitonic contributions to the Faraday rotation (see Fig. 1b). To study the validity of our model in the regime $E / E_{\text {gap }}<0.98$, i.e. when the photon energy $E=\hbar \omega$ is smaller than the band-gap energy $E_{\text {gap }}$, we also consider the Faraday rotation in a thicker $\mathrm{Cd}_{0.9} \mathrm{Mn}_{0.1}$ Te sample $[3,5]$.

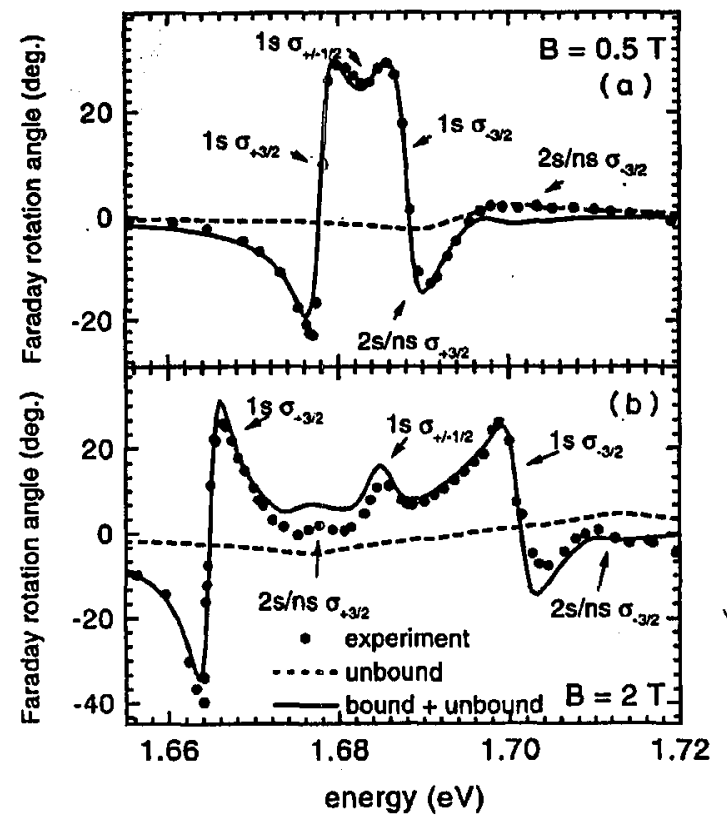

Fig. 1. Linear Faraday rotation angle for the $\mathrm{Cd}_{0.95} \mathrm{Mn}_{0.05}$ Te layer at $5 \mathrm{~K}:$ (a) $B=0.5 \mathrm{~T}$ and (b) $B=2 \mathrm{~T}$. Experimental points are denoted as circles and theoretical results as dashed/straight lines corresponding to unbound/bound+unbound contributions.

To model all data within only one set of optical parameters, it is of crucial importance to incorporate the strong Coulomb enhancement of bound and unbound transitions of Wannier excitons [6,7] in II-VI compound semiconductors. The excitonic effects are included in the microscopic model of Ref. [5] by replacing the dipole matrix-element $d_{0}$ for each optical transition by $d=d_{0}\left|f_{n, k}(r=0)\right|^{2}$, with $f(r=0)$ - the amplitude of the excitonic wave function at the relative electron-hole coordinate $r=0$ [7]. Thus we can calculate the imaginary part of the index of refraction and thereby the transmission trace for zero field. Fitting the amplitude of the transmission trace, we deduce the Rydberg of $11.5 \mathrm{meV}$ (or $a_{\mathrm{B}}=67 \AA$ ) and the dipole matrix-element for the optical transition $d_{0}=e \times 5.6 \AA$ (corresponding to $E_{\mathrm{P}}=23 \mathrm{eV}$ ).

The exchange-induced energy splitting of the excitons with the magnetic field is generally set equal to the splitting of the valence- to conduction-band transitions at $k=0$, thus neglecting the $k$-dependence for all excitonic transitions. 
This approximation is valid for all Rydberg excitons, if the wave vector spread of the excitonic wave function in $k$-space is small enough, i.e. the exchange decay $k_{0}$ in $k$-space is slow enough: $k_{0} \gg 1 / a_{\mathrm{B}}=1.5 \times 10^{8} \mathrm{~m}^{-1}$. This condition is fulfilled, if we consider the value obtained theoretically $\left(k_{0} \approx 2 \times 10^{9} \mathrm{~m}^{-1}\right.$, Ref. [8]) or for the value we deduced in Ref. [3] $\left(k_{0}=1.3 \pm 0.3 \times 10^{9} \mathrm{~m}^{-1}\right)$ but not for that one used in Ref. [5] $\left(k_{0}=2.6 \times 10^{8} \mathrm{~m}^{-1}\right)$.

The results of our calculations [3] are depicted in Fig. 1. Even with the Coulomb enhancement of the unbound interband transitions taken into account, the resulting Faraday rotation in the bound excitonic region is ten times smaller. Increasing the field up to $2 \mathrm{~T}$, the peak positions of all excitonic transitions are in good agreement with theory, see Fig. 1b. Note that the observation of the same exchange-induced shift for the $1 s$ and $2 s / n s \sigma_{ \pm 3 / 2}$ transitions is consistent with our larger value of $k_{0}$.

To study the impact of the Coulomb interactions on the photoinduced Faraday rotation we increased the laser fluence in the transmission geometry. The linear and photoinduced Faraday rotation spectra at $B=0.5 \mathrm{~T}$ are depicted in Fig. 2. At about $1 \mu \mathrm{J} / \mathrm{cm}^{2}$ the nonlinear regime sets in and the Faraday rotation spectra

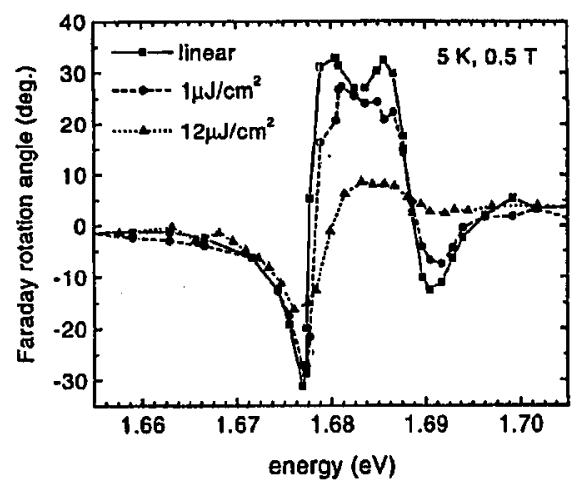

Fig. 2. Nonlinear Faraday rotation angle for the $\mathrm{Cd}_{0.95} \mathrm{Mn}_{0.05}$ Te layer at three different excitation densities at $5 \mathrm{~K}$ and $0.5 \mathrm{~T}$.

undergo drastic modifications: photoinduced changes of the Faraday rotation angle of up to $25^{\circ}$ at fluences as low as $100 \mathrm{fJ} / \mathrm{cm}^{2}$ can be detected. A strong saturation of the excitonic transitions and a spectral blue shift are observed.

To model this experimental results within our microscopic model for the linear Faraday rotation, only the dominant $1 s$ excitonic transitions are considered. The effects of photoinduced excitonic nonlinearities due to a number $N$ of optically excited electron-hole pairs are accounted for in the first order by a density dependent saturation $f=f\left(1-N / N_{f}\right)$, screening effects $E_{\mathrm{G}}=E_{\mathrm{G}}\left(1-N / N_{\mathrm{G}}\right)$, exchange interaction $E_{\mathrm{R}}=E_{\mathrm{R}}\left(1-N / N_{\mathrm{R}}\right)$ and spectral broadening $\Gamma=\Gamma\left(1-N / N_{\Gamma}\right)$, see e.g. Refs. [9-11]. Careful fitting of our data reveals a spectral blue shift of 1-2 meV, i.e., a resulting blue shift in a $3 \mathrm{D}$ bulk CdMnTe at the same order as in 2D CdTe/CdMnTe quantum wells [1]. 
This is in strong contrast to the excitonic nonlinearities in bulk GaAs. Here the renormalization of the excitonic binding energy (blue shift) and the band-gap renormalization (red shift) cancel each other in the first approximation. The energy position of the $1 s$ exciton is unchanged even at high excitation densities below the Mott density, see e.g. Ref. [11]. Generally, this is attributed to the charge neutrality of the exciton. The energy shifts of the screening effects and the exchange interaction cancel each other in the first-order corrections. As the excitonic binding energy in our bulk CdMnTe system $\left(E_{\mathbf{R}}=11.5 \mathrm{meV}\right)$ is considerably larger than for GaAs (4.2 meV), i.e., the Bohr radius is smaller, higher order contributions might have to be taken into account due to the strongly increased Mott density [9].

In conclusion, we presented experimental results on the linear and photoinduced Faraday rotation in a high quality MBE grown $\mathrm{Cd}_{1-x} \mathrm{Mn}_{x} \mathrm{Te}$ sample. The absolute angle of the linear Faraday rotation was calculated using a microscopic model for the complex dielectric constant. To explain the identical shift of $1 s$ and $2 s / n s \sigma_{ \pm 3 / 2}$ excitons at $5 \mathrm{~K}$ and the absolute amplitude of the interband contribution, a $k$-dependence of the magnetic spin-exchange interaction described by a decay constant $k_{0}=1.3 \pm 0.3 \times 10^{9} \mathrm{~m}^{-1}$ is required. All optical parameters are in excellent agreement with theoretical predictions. At low temperatures and close to the band-edge the $1 s$ excitonic contribution is the most important for linear and photoinduced Faraday rotation. Due to the strong Coulomb enhancement of the $1 s$ bound excitonic transition, photoinduced Faraday rotations of up to $25^{\circ}$ at fluences of only $100 \mathrm{fJ} / \mathrm{cm}^{2}$ are observed. The high excitonic binding energy of $11.5 \mathrm{meV}$ leads to strong excitonic nonlinearities, resulting in a net blue shift of 1-2 $\mathrm{meV}$ of the $1 s$ transition.

P. Leisching gratefully acknowledges financial support by the Alexander von Humboldt foundation.

\section{References}

[1] C. Buss, R. Pankoke, P. Leisching, J. Cibert, R. Frey, C. Flytzanis, Phys. Rev. Lett. 78, 4123 (1997).

[2] J.A. Gaj, R.R. Galązka, M. Nawrocki, Solid State Commun. 25, 193 (1978); J.A. Gaj, J. Ginter, R.R. Gałązka, Phys. Status Solidi B 89, 655 (1978).

[3] P. Leisching, R. Pankoke, C. Buss, R. Frey, C. Flytzanis, J. Cibert, A. Wasiela, Phys. Rev. B 54, R8305 (1997).

[4] D.U. Bartholomew, J.K. Furdyna, A.K. Ramdas, Phys. Rev. F 34, 6943 (1986).

[5] C. Buss, S. Hugonnard-Bruyère, R. Frey, C. Flytzanis, Solid State Commun. 92, 929 (1994); S. Hugonnard-Bruyère, C. Buss, F. Vouilloz, R. Frey, C. Flytzanis, Phys. Rev. B 50, 2200 (1994).

[6] C. Buss, R. Frey, C. Flytzanis, J. Cibert, Solid State Commun. 94, 543 (1995).

[7] R.J. Elliott, Phys. Rev. 108, 1384 (1957).

[8] A.K. Bhattacharjee, in: Proc. 20th Int. Conf. on Physics of Semiconductors, Vol. 1, Eds. E.M. Anastassakis, J.D. Yoannopopoulos, World Scientific, Singapore 1990, p. 763.

[9] P. Pankoke, P. Leisching, C. Buss, R. Frey, C. Flytzanis, unpublished.

[10] S. Schmitt-Rink, D.S. Chemla, D.A.B. Miller, Phys. Rev. B 32, 6601 (1985).

[11] H. Haug, S. Schmitt-Rink, Prog. Quant. Electron. 9, 3 (1984). 